Revue des patrimoines

\title{
Chevaux de travail du XIXe siècle : à la recherche des éleveurs de l'Orne
}

\section{Agnès Manneheut}

\section{(2) OpenEdition}

1 Journals

\section{Édition électronique}

URL : http://journals.openedition.org/insitu/12193

DOI : $10.4000 /$ insitu. 12193

ISSN : 1630-7305

Éditeur

Ministère de la Culture

\section{Référence électronique}

Agnès Manneheut, "Chevaux de travail du XIXe siècle : à la recherche des éleveurs de I'Orne », In Situ [En ligne], 27 | 2015, mis en ligne le 28 octobre 2015, consulté le 10 décembre 2020. URL : http:// journals.openedition.org/insitu/12193; DOI : https://doi.org/10.4000/insitu.12193

Ce document a été généré automatiquement le 10 décembre 2020.

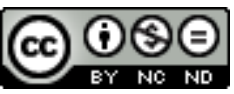

In Situ Revues des patrimoines est mis à disposition selon les termes de la licence Creative Commons Attribution - Pas d'Utilisation Commerciale - Pas de Modification 4.0 International. 


\title{
Chevaux de travail du XIXe siècle : à la recherche des éleveurs de l'Orne
}

\author{
Agnès Manneheut
}

\section{Introduction}

1 En matière d'élevage, le $\mathrm{XIX}^{\mathrm{e}}$ siècle fut une période de mutations avec la définition et la spécialisation de races animales domestiques et, en parallèle, l'émergence des éleveurs. Nouvelle catégorie du monde agricole, ceux-ci sélectionnent les reproducteurs, mâles et femelles, les gardent ou les vendent comme tels, font naitre de jeunes sujets et en achètent d'autres; certains firent ainsi du cheval une production à part entière de leur exploitation. En mettant en place cet élevage "complet», ils se distinguaient tant des paysans "naisseurs", utilisateurs de juments et vendeurs des poulains mâles, que des cultivateurs des plaines céréalières ou " pays d'élevage », qui achetaient ces jeunes mâles pour les mettre au travail à la charrue avant de les revendre, dressés, musclés et prêts à servir, notamment dans les transports ${ }^{1}$. Ces mutations sont particulièrement visibles dans le département de l'Orne, où se côtoient quatre régions agricoles distinctes, ayant chacune son système d'élevage, et participant des trois grands systèmes agraires normands: le Perche avec son agriculture polyvalente et ses massifs forestiers, le pays d'Auge ornais aux herbages plantureux, les plaines centrales à dominante céréalière d'Alençon à Argentan, et, à l'ouest, le Bocage aux sols pauvres, isolé et fermé2.

2 Un vaste patrimoine écrit témoigne de ces transformations, lié à l'existence d'institutions spécialisées et pérennes, à la place centrale du cheval dans la vie quotidienne et notamment agricole, mais aussi à son rang symbolique dans les représentations sur l'élevage, l'agriculture ou plus largement la société.

3 Les préoccupations premières portaient sur le cheval de guerre, et la place de choix dans les écrits comme dans les représentations picturales est tenue par le cheval de course, à la mesure du rôle des hippodromes dans l'amélioration des races. Qu'en est-il du patrimoine lié à l'élevage des chevaux que l'on pourrait dire «de travail », dont la production était 
étroitement liée à un contexte agricole, et non à des capitaux extérieurs et méthodes importées? La dénomination désigne en particulier le percheron, qui a fait couler beaucoup d'encre dès la Restauration, et d'autres animaux relevant de types de trait plus ou moins lourd, mais elle concerne également des chevaux un peu plus fins, qui au début de la période n'étaient pas encore appelés « de demi-sang » et qui étaient employés à la culture dans les plaines aux terres légères 3 .

\section{Des institutions très présentes}

\section{Les archives de l'Administration des haras}

Versées en 2005 aux archives départementales de l'Orne, les archives produites par le prestigieux haras du Pin, situé au cœur du département, sont loin d'avoir été exploitées autant qu'elles le mériteraient. Elles sont d'autant plus précieuses que les dossiers de l'Inspection des haras conservés aux Archives nationales, qui comprennent notamment des rapports d'inspection et des états de saillie d'étalons approuvés, ne couvrent pas la période comprise entre les années 1840 et 1870.

5 L'intérêt premier des archives du dépôt d'étalons est bien sûr d'apporter une vision globale de la population équine locale, au travers des rapports sur l'état de l'élevage dans la circonscription envoyés annuellement par le directeur à son ministère de tutelle ${ }^{4}$. Cette source aborde l'évolution des conditions d'élevage aussi bien que les événements qui l'affectent, comme les aléas agricoles ou les tendances du commerce, et c'est l'une de celles qui nous décrit l'emploi de jeunes chevaux de demi-sang dans certaines plaines aux terres légères. S'y ajoutent une correspondance dépeignant les affaires du monde hippique, des rapports sur les courses et concours et des rapports sur les saisons de monte. Ceux-ci nous livrent quelques détails sur des habitudes locales : par exemple, les fermiers des alentours de Flers ramenaient leur jument quatorze jours après la première saillie, et non neuf comme ceux de la plaine d'Écouché5. Ils décrivent surtout les grandes lignes des choix des éleveurs, notamment les tendances générales vers des modèles plutôt forts ou au contraire allégés, selon les années.

Pour suivre plus particulièrement ces choix dans un lieu et sur une période donnés, ou évaluer l'influence des reproducteurs, voire reconstituer des lignées, il est possible de recourir aux registres de description des étalons et aux feuilles et cartes de saillies ${ }^{6}$. C'est ainsi que l'on peut chercher les traces de la descendance de deux étalons orientaux, Godolphin et Gallipoly, cités par plusieurs ouvrages comme les géniteurs ayant " régénéré » la race percheronne avec du sang arabe après les guerres napoléoniennes, et lui ayant transmis une robe gris pommelé 7 . Or, le premier est décrit dans les registres du haras comme bai et de parents anglais, et s'avère n'avoir sailli que huit juments lors de son unique année de monte dans le Perche ornais, produisant quatre poulains ; Gallipoly, quant à lui, ne fut jamais envoyé dans une station percheronne, mais effectua toutes ses saisons de monte au haras ${ }^{8}$.

Il est à noter que les registres ne concernent pas seulement les étalons du dépôt : depuis l'Ancien Régime, l'administration des haras a cherché à surveiller les chevaux entiers des particuliers, de manière à influencer l'amélioration des races plus largement que par ses propres géniteurs. À partir de 1820, deux catégories d'étalons privés furent ainsi définies : ceux que l'administration jugeait propres à améliorer les races étaient "approuvés » et recevaient des primes après la saison de monte, s'ils avaient sailli un nombre minimal de 
juments. Ceux qui étaient moins bons mais exempts de défauts majeurs ou de tares héréditaires étaient simplement " autorisés »; leurs propriétaires ne touchaient aucune rémunération mais avaient accès à la clientèle des juments destinées à participer à des concours. Dans les faits, les éleveurs et les étalonniers, voire quelques fonctionnaires de la préfecture, mirent plusieurs années à maîtriser la subtilité des dénominations, droits et obligations liés. En août 1885, pour contrôler entièrement les étalons servant à la reproduction et éradiquer certaines maladies héréditaires, une troisième catégorie fut instaurée, celle des « acceptés » ou « admis » : il était désormais interdit d'utiliser pour la monte publique un animal n'ayant pas été examiné et agréé par une commission sanitaire.

8 Ce système de délégation à l'étalonnage privé était d'autant plus important pour les chevaux de travail que l'administration des haras fournissait très peu d'étalons de trait : il était facile de se reposer sur des particuliers pour leur entretien, tandis que celui de chevaux de sang nécessitait un savoir-faire moins répandu et, du fait qu'ils ne travaillaient pas, des moyens financiers plus importants.

Bien sûr, cette surveillance ne nous permet de connaître la totalité des reproducteurs qu'à partir de 1885 : la plupart des cultivateurs, n'ayant pas besoin de justifier les origines de leurs poulains, cherchaient la saillie la moins chère ou la plus proche, et recouraient à un cheval de meunier, à un poulain de deux ans vendu après la saison de monte ou à un étalon « rouleur » non répertorié, se déplaçant de ferme en ferme, ou encore à l'entier de quelqu'un avec qui ils avaient affaire par ailleurs.

10 Ce contrôle permet néanmoins, via la description de chaque cheval dûment consignée dans les registres du haras et les listes publiées chaque année, d'identifier les étalonniers et de repérer les caractéristiques de leurs écuries telles le nombre et le type des étalons, ou la durée de service de chaque cheval. Il est à noter qu'à la fin du XIX ${ }^{\mathrm{e}}$ siècle, les reproducteurs percherons des élevages les plus reconnus ne travaillaient plus : un éleveur de chevaux d'excellence peut se passer de leur force de travail. Les besoins diminuaient du fait que dans ces exploitations, les herbages prenaient le pas sur les labours; confier les travaux restants à des chevaux plus communs permettait de limiter les risques de blessure pour des étalons de grande valeur, mais surtout, sur le plan symbolique, de ne pas les faire déroger, et de marquer l'aisance du propriétaire qui peut entretenir des animaux inactifs (fig. 1). 
Figure 1

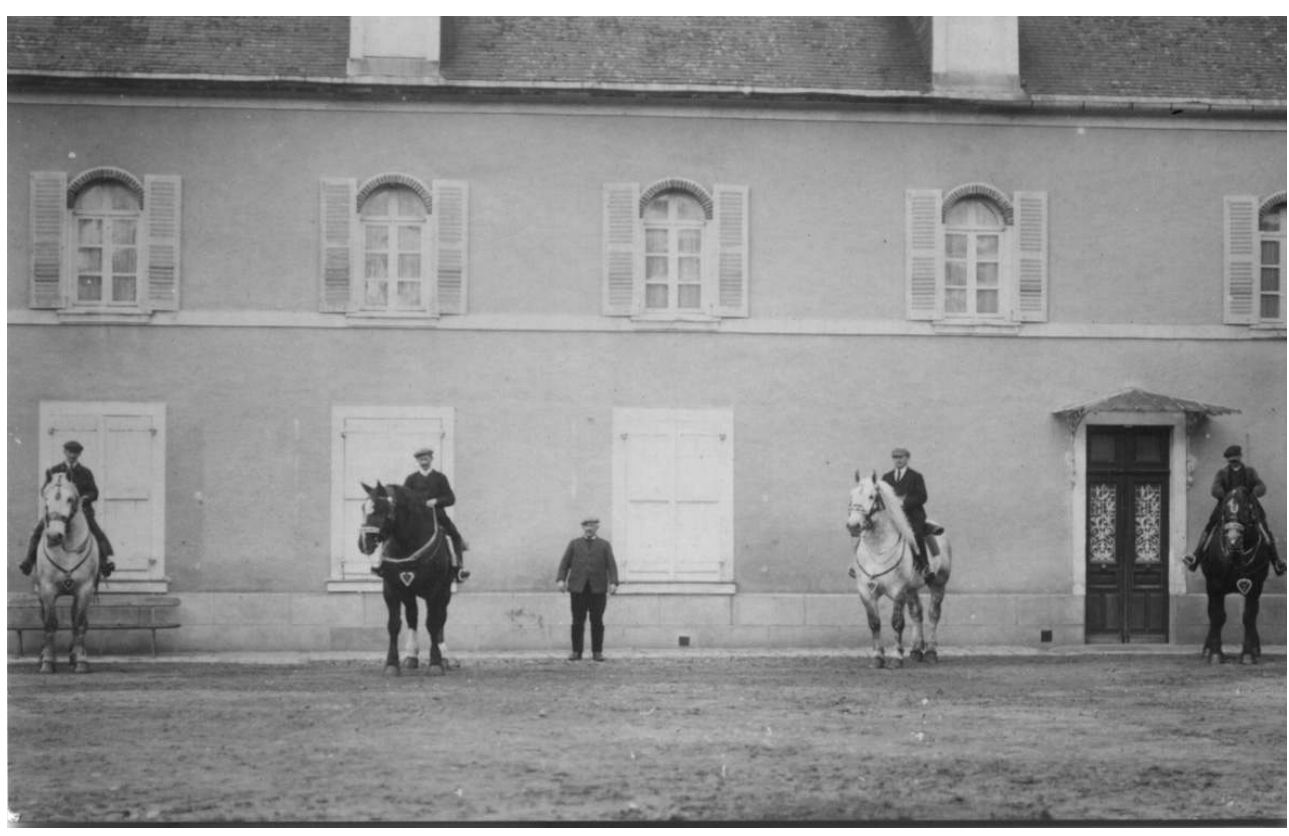

«Conducteurs » des étalons de M. Aveline sur le départ, à Verrières. Carte postale, début du Xxe siècle. (C) Écomusée du Perche.

De plus, pour obtenir la prime, les propriétaires d'étalons approuvés devaient transmettre à l'administration les listes des juments servies par chaque cheval ; ces états de saillie n'ont hélas été conservés que pour les années 1820 à 1836, aux Archives nationales et pour certains également aux archives départementales de l'Orne. Ils font apparaître la clientèle des propriétaires de poulinières, avec leur mobilité géographique et leur fidélité à un fournisseur9

12 Enfin, à l'instar de celles d'autres dépôts d'étalons, les archives du Pin pourraient également susciter diverses recherches, notamment une prosopographie des agents, d'autant que le haras héberge l'École des haras, ou une étude de l'économie du domaine.

\section{Les papiers de l'administration des remontes militaires}

13 Établi progressivement à partir de la Restauration, le service des remontes militaires avait pour but principal d'acheter des chevaux adaptés aux besoins de l'armée, en s'appuyant sur l'expertise d'officiers spécialisés ; il devait aussi influencer indirectement la qualité de l'élevage, par le conseil aux éleveurs et par l'encouragement financier d'une juste rémunération. Sur ce plan, son action était donc complémentaire de celle des haras, qui œuvraient à l'amélioration de la production pour les besoins militaires de manière directe, en fournissant des géniteurs de qualité. En pratique, les avis des deux administrations sur le bon cheval de guerre ont parfois été bien divergents, et les polémiques n'ont pas manqué sur l'opportunité de donner également aux militaires la possibilité d'agir directement sur la production; enfin, une certaine lutte d'influence auprès des éleveurs a pu se ressentir au moment de la vente des meilleurs jeunes mâles.

Le recours à cette source est incontournable pour étudier l'élevage des chevaux de selle car les achats militaires ont été très structurants jusqu'à la Première Guerre mondiale. 
Situé dans un département d'élevage " de luxe », le dépôt d'Alençon, tantôt établissement de plein exercice, tantôt succursale de celui de Caen, fournissait surtout des chevaux d'officier et se distinguait par la qualité de ses achats. Dans l'Orne, la majorité des acquisitions n'étaient donc pas des chevaux de travail, mais des produits destinés à la carrière d'étalon, à défaut vendus à une clientèle de particuliers aisés ; la vente à l'armée, dont les moyens étaient souvent inférieurs, n'était pas le débouché visé, même si elle assurait un écoulement régulier de la production. Néanmoins, au cours de ses tournées fréquentes dans la circonscription, la commission trouvait aussi à acheter un certain nombre de chevaux de troupe, demi-sang des fermes, et des chevaux de trait léger pour l'artillerie.

15 Le service n'ayant pas survécu à la motorisation des armées, les archives conservées sont moins riches et structurées que celles des haras. Il s'agit principalement de dossiers versés au Service historique de la Défense (SHD), notamment d'irremplaçables rapports mensuels de tournées d'exploration pour les années 1862-1875, complétés par quelques documents des archives départementales de l'Orne ${ }^{10}$.

Ces rapports sont à mettre en relation avec plusieurs sources imprimées. À de nombreuses reprises en effet se sont exprimées des réflexions sur l'action des remontes et leur contribution à la défense nationale, dans des brochures parfois polémiques. Mais le complément essentiel aux documents écrits au sein du service des remontes se trouve dans les recueils des actes administratifs des départements : à partir de 1850 fut instaurée la publication officielle des procès-verbaux de réception des chevaux achetés, qui nous fournit des listes entières d'animaux avec leurs âge, taille, robe, classement, c'est-à-dire l'arme à laquelle ils convenaient de par leur conformation, bien sûr le nom de leur vendeur et sa commune, et enfin leur prix d'achat. L'objectif était d'assurer les éleveurs que l'armée ne payait pas les chevaux en-dessous des prix du commerce, pour les encourager à amener leurs produits à la commission de remonte. La comparaison avec les statistiques d'achats conservées à Vincennes (SHD) montre que ces listes ne sont pas aussi exhaustives qu'elles étaient censées l'être, mais elles n'en fournissent pas moins des séries de données considérables, qui permettent notamment de cerner la figure essentielle et ambiguë du marchand.

17 Celui-ci apparait en effet comme un rouage central du commerce, tout aussi indispensable aux cultivateurs, à qui il offrait un débouché immédiat et un paiement en espèces sonnantes et trébuchantes, qu'aux officiers du dépôt qui ne pouvaient remplir leurs commandes avec les ressources amenées par les seuls éleveurs. Pourtant, les marchands furent exclus à plusieurs reprises du marché militaire, en tant qu'intermédiaires "parasites» qui privaient les petits éleveurs d'une partie de leur bénéfice légitime, en tant que vendeurs trop habiles dans l'art de mettre en condition des chevaux et de se les faire payer au-dessus de leur valeur, et en tant que patriotes peu scrupuleux, susceptibles d'importer des chevaux d'Allemagne pour les vendre à l'armée au détriment de la production locale. L'« achat direct » à l'éleveur semble ainsi avoir été la règle de 1837 à 1847 , de 1853 à 1856, de 1858 à 1861, de 1871 à 1897 ; la fréquence de ces changements de législation témoigne du caractère incontournable des marchands mais aussi de la difficulté à circonscrire précisément cette catégorie. Le principal critère défini pour écarter leurs chevaux était la durée de détention: le vendeur devait produire un certificat du maire de sa commune attestant que l'animal était en sa possession depuis plus de six mois. La géographie fut également prise en considération entre 1861 et 1870, l'interdiction étant alors levée uniquement pour les marchands et les produits de la 
circonscription, et à partir de 1897, une pondération se fit sur le volume d'activité du vendeur : les personnes amenant moins de vingt chevaux furent dispensées de produire un certificat. La porosité entre les activités de marchand et d'éleveur est ainsi manifeste : tous deux achètent des produits pour les revendre, le premier souvent sur une échelle plus large du fait que c'est son activité principale, tandis que le second acquiert des sujets relativement jeunes, voire en fait naître quelques-uns, et les fait plus ou moins dresser. Le Recueil des actes administratifs désigne d'ailleurs parfois un vendeur, à la rubrique "profession", comme "éleveur-marchand", confirmant ainsi qu'en pratique, «en Normandie, les rôles de l'éleveur et du marchand se confondent et [qu']il est impossible, dans cette confusion, de distinguer nettement là où s'arrête l'un et commence l'autre ${ }^{11}$ ». Les procès-verbaux de réception montrent également que les grossistes faisant voyager des chevaux à travers la France, principale cible de l'exclusion, n'étaient guère vendeurs dans l'Orne en temps de paix: les marchands qui bénéficiaient du marché militaire y étaient bien des intermédiaires locaux, fins connaisseurs de l'offre comme des exigences de l'armée, avec lesquels les officiers pouvaient établir des relations sur moyen ou long terme, gage de qualité.

\section{L'intérêt marqué des institutions locales}

18 Le $\mathrm{XIX}^{\mathrm{e}}$ siècle a vu fleurir les encouragements à un élevage chevalin menacé de dégénérescence, concurrencé par celui des animaux de boucherie, et pour le cheval de selle, par celui de trait, ou par l'import de chevaux étrangers. À cet effet, des primes étaient distribuées par catégorie de sexe, d'âge et d'espèce; certaines étaient liées à des épreuves de course au trot ou de dressage, destinées à récompenser les chevaux sur leurs performances ou du moins leurs capacités, et non sur leur seule conformation. Dans l'Orne, dès le Premier Empire, préfet et conseil général se sont impliqués dans la mise en place de concours d'élevage ${ }^{12}$. Des discussions, voire des débats, à propos du budget à leur allouer et plus généralement, de « l'industrie chevaline » émaillaient donc régulièrement les séances de l'assemblée départementale. Il s'agissait de participer à la qualité de l'élevage, malmené par les guerres révolutionnaires et impériales, et d'améliorer tant la race des percherons que celle des demi-sang, d'abord par croisement puis par sélection; plus largement, l'action visait à la prospérité locale et aux progrès agricoles. Dans les procès-verbaux des séances s'expriment notamment des opinions sur l'action de l'administration des haras et son évolution, fournissant un contrepoint aux rapports émanant de cette institution. Les discours tenus permettent également d'aborder la géographie de l'élevage, à travers les souhaits de voir créer de nouvelles stations de monte ou modifier la répartition des étalons.

19 Les concours se révèlent également être une mine d'informations grâce à leurs palmarès, qui permettent de repérer de nombreux éleveurs, notamment les propriétaires de poulinières. À la fin du siècle, l'éventail des épreuves allait du comice cantonal au concours général agricole à Paris, en passant par les comices d'arrondissement nés dans les années 1840, les primes départementales ou le concours agricole régional. Quel que soit le niveau observé, les comptes rendus de ces réunions ne permettent d'appréhender que la frange supérieure de chaque catégorie d'éleveurs, ceux qui s'investissaient dans la sélection et présentaient les produits de leur exploitation sans peur, voire avec fierté ${ }^{13}$.

La majorité des primes était destinée aux chevaux de demi-sang et les récompenses allaient aux animaux d'herbage plus que de travail ; une exception notable est celle du 
concours pour «poulinières de demi-sang employées aux travaux de l'agriculture » créé en 1873 à Écouché ${ }^{14}$. Les courses n'étaient pas non plus réservées aux chevaux de selle : dès 1839 , la Société hippique d'Alençon organisa quelques épreuves pour chevaux percherons, ainsi que celle de Mortagne à partir de $1851^{15}$. Mais ce sont surtout les comices agricoles qui, au plus près du terrain, ont consacré des concours aux chevaux de trait. Fondés avec le soutien des institutions locales, d'abord au niveau des chefs-lieux d'arrondissement puis à celui des cantons, certains de ces comices ont publié des annuaires très intéressants; malheureusement leur diffusion inégale et leur fragilité n'ont pas toujours permis d'en conserver des séries complètes. Pour les années manquantes et pour les autres rassemblements, heureusement, les palmarès ont souvent été conservés à la préfecture pour justifier une subvention, et parfois repris par la presse locale.

21 Ces palmarès permettent de voir que certains propriétaires présentaient aux jurys uniquement des juments ou des mâles, d'autres faisaient cohabiter les deux sexes; d'autres encore cherchaient l'excellence dans leurs diverses productions, bovins, ovins, cidres, ou encore dans le concours de l'exploitation la mieux tenue. Les comptes rendus des visites de fermes effectuées dans le cadre de cette dernière catégorie ont parfois été publiés dans un annuaire, fournissant un instantané sur l'état d'une exploitation accompagné d'un commentaire qualitatif sur les choix de culture ou la construction et l'entretien des bâtiments. Pour ne concerner que d'assez grandes fermes peu représentatives de la moyenne, ces rapports n'en sont pas moins très instructifs.

À côté des affiches et de la correspondance relative à ces diverses épreuves, les archives départementales conservent d'autres traces de courriers entre le préfet et de grands éleveurs ou des autorités locales, par exemple à propos d'acheteurs supposés allemands et soupçonnés d'écumer nos campagnes du fleuron de leur production. Enfin, apparaît une partie de l'activité des écoles de dressage, où l'éducation d'animaux de valeur était parachevée pour les vendre à des particuliers aisés recherchant des produits "prêts à servir ».

23 Au niveau local, les communes ont parfois conservé des documents intéressants produits sur demande de l'État, notamment les enquêtes agricoles et communales ${ }^{16}$. En 1873 en particulier fut instauré un recensement exhaustif des chevaux et des mules dans une perspective militaire, saisi à partir de 1877 dans des formulaires spécifiques et mis à jour pendant plusieurs années. Il se déroulait en trois étapes: un premier registre de "déclaration" recensait tous les chevaux, le deuxième, dit "de recensement", ne retenait que ceux âgés de plus de six ans et non réformés, et le dernier classait par arme ces animaux mobilisables. Dans l'Orne, deux communes ont conservé le registre de déclaration, donnant une photographie à un instant précis de la population chevaline, notamment les répartitions selon le sexe et selon l'âge.

\section{Retrouver les acteurs principaux}

Quelques grands éleveurs ont directement pris la plume pour s'adresser au préfet ou à un député ; la plupart sont concernés par la production du cheval de luxe, non de celui qui travaille, et ils font partie d'une élite peu représentative du commun des producteurs.

D'autres ont très probablement laissé des traces dans des papiers familiaux, notamment parmi les dynasties fondatrices de la race percheronne; des collections de plaques de 
concours existent peut-être encore dans certaines fermes. Il n'est pas impossible non plus que des mémoires familiaux ou des cahiers de comptes mentionnant le cheval, conservés ici et là, entrent un jour dans des collections publiques au cours d'une campagne de collecte sur un autre thème ou à l'occasion d'événements privés. Enfin, l'architecture liée à l'élevage est également difficile à retrouver, du fait qu'il se faisait en grande partie en plein air, et que les mutations de l'agriculture ont depuis considérablement transformé les bâtiments. Néanmoins, quelques écuries d'excellence ont pu être conservées, voire protégées, comme celles de la Ferme neuve, située dans la commune de Dorceau.

La parole individuelle des éleveurs est donc rare, mais ils se sont aussi organisés collectivement en associations pour porter un discours commun. La Société hippique percheronne de France, fondée en 1883, est la seule à exister encore aujourd'hui. Ses archives, avec la tenue du stud-book, ne concernent pas seulement les chevaux et leurs lignées mais aussi des orientations collectives et probablement des choix individuels: adhérer à une telle association consiste à faire reconnaître ses juments mais conduit aussi à se positionner dans une race et dans son amélioration, et aide à s'insérer dans des circuits commerciaux.

Avant la fondation officielle de la race percheronne, et pour les autres espèces, il faut interroger d'autres sources pour aborder les producteurs de chevaux de travail : à partir des noms d'éleveurs transmis par les sources citées ci-dessus, il est possible de sélectionner des échantillons de marchands, d'étalonniers ou de cultivateurs utilisant des juments, et de chercher leurs traces dans les archives d'état civil, notariales, ou encore judiciaires. Ainsi, le malheur d'Alfred Bignon, membre d'une dynastie d'éleveurs primés des communes de Bures et La Mesnière, orphelin de père à six ans en 1880 et de mère l'année suivante, fait le bonheur de l'historien: le premier inventaire après décès énumère tous les équipements de la ferme liés au travail du cheval, à savoir un attelage de limon complet, une selle de limon, une couverture d'attelage, une charrue avec son train, quatre colliers dont un à charroyer et un à labourer, deux paires de traits et un cabriolet. Il détaille bien sûr aussi le cheptel, son âge et sa valeur: dans cette exploitation d'une bonne vingtaine d'hectares principalement en labours, les quatre juments côtoyaient trois vaches avec leurs veaux, un veau plus âgé, six bœufs, quatre porcs et une brebis avec ses agneaux ${ }^{17}$. On peut d'ailleurs remarquer qu'une des juments de trois ans était déjà suitée $^{18}$ : les efforts des officiers des haras n'avaient pas encore suffi à éradiquer la saillie des pouliches de deux ans. L'inventaire de 1881 révèle qu'après le décès de son mari, Mme Bignon vendit l'ensemble des bêtes en une vente mobilière ${ }^{19}$.

Une utilisation approfondie des archives notariales, en particulier des baux ruraux, qui dans certaines régions semblent avoir limité l'entretien de chevaux à un pour dix bovins, permettrait ainsi de mieux appréhender la place du cheval dans les patrimoines et surtout dans l'espace et dans les systèmes agraires ${ }^{20}$.

\section{Des spectateurs intéressés}

\section{Les représentations figurées}

Jusqu'à l'apparition de la photographie, l'iconographie est également difficile à interpréter : en particulier, les dessins de chevaux percherons parus dans le Journal des haras semblent refléter plus l'opinion du commanditaire du dessin sur le bon cheval que 
la réalité des conformations. En revanche, les représentations photographiques peuvent être riches de détails, comme en témoigne, pour la même espèce, la très belle collection de cartes postales rassemblée par l'écomusée du Perche.

\section{Des discours savants}

31 Agronomes, zootechniciens et vétérinaires n'ont pas manqué de s'exprimer tout au long du siècle, dans des ouvrages scientifiques ou des manuels pratiques, sur les chevaux en général, sur leur rôle dans l'agriculture ou sur telle ou telle race en particulier. Cet arrière-plan théorique renseigne sur les conditions de l'élevage, voire sur le rapport à l'animal. Ainsi le rapport d'un vétérinaire ornais surpris par la grosseur d'un calcul urinaire nous décrit la réaction d'un cultivateur à une morsure : la peur de la rage lui fit donner un grand coup de poing sur la tête de la jument, qui « se mit à trembler très fort " ${ }^{21}$.

Mais cette littérature savante représente une masse très volumineuse et parfois difficile à appréhender en raison du caractère théorique de certains discours, notamment ceux des zootechniciens. Connaître ce fond idéologique permet d'interpréter d'autres sources, par exemple les rapports des directeurs des haras, souvent influencés par la doctrine et ses jugements de valeur. Mais il faut prendre garde qu'un même vocabulaire peut de ce fait désigner des réalités différentes selon qu'il est utilisé sur le terrain ou dans des traités, par des militaires, des savants ou des personnalités locales. Cas emblématique, l'expression " cheval commun " peut correspondre selon le contexte à un jeune mâle très bon mais manquant trop de distinction pour faire un étalon, ou à une bête sans caractéristiques particulières ni beaucoup de valeur, d'un type très répandu.

$\mathrm{Au}$ croisement des considérations savantes et de la parole locale, l'Association normande d'Arcisse de Caumont s'impliquait beaucoup dans les progrès de l'agriculture, sans y être spécialisée. Elle se réunissait chaque année dans un canton différent des «cinq départements » et l'intérêt de son annuaire réside en particulier dans la description de la situation agricole de la contrée visitée, ainsi que dans ses débats alimentés par des points de vue locaux sur les réorganisations de l'administration des haras ou des remontes militaires.

\section{La presse locale}

La fréquence de mentions relatives au cheval ou à un cheval dans les journaux du département reflète le poids symbolique de cet animal et l'importance de sa présence quotidienne. La presse se faisait en effet l'écho d'avis sur les races ou sur les étalons des haras ; en 1853 notamment, tout un échange sur l'avenir du percheron fut publié par l' Écho de l'Orne.

On trouve également dans les quotidiens ou hebdomadaires des comptes rendus de courses, de comices agricoles, et surtout de foires, permettant de suivre les fluctuations du commerce, années fastes avec marchandise de qualité et profusion d'acheteurs ou rassemblements médiocres à cause du mauvais temps. Il y paraissait enfin des annonces et la mention de faits divers plus ou moins anodins: vols ou retrouvailles d'animaux volés, décès ou blessures liés à des ruades ou des emballements, mais aussi compte rendu du procès en assises d'un marchand de chevaux accusé d'en avoir assassiné un autre. Du côté des incidents cocasses, on peut citer la bévue d'un cultivateur de Courgeon qui en 
1887 ramena de la foire de Mortagne le poulain d'un autre et ne se rendit compte de sa méprise qu'une fois arrivé chez lui, à quelques kilomètres de distance, « d'autant plus vite que le poulain n'était pas du même sexe $»^{22}$.

Ces faits divers nous donnent aussi un aperçu de la frange inférieure de la population équine, par ailleurs peu visible même si elle apparait dans les rares registres conservés de déclaration pour le recensement, ou si la plume d'un directeur de haras évoque, au détour de la description de la population équine d'une contrée, des juments «peu susceptibles d'amélioration ", des bidets sans intérêt ou, au degré le plus bas de la hiérarchie, les chevaux de charbonnier des massifs forestiers. Ces animaux de misère, vieux et usés, ou sans race définie, trimaient plus qu'ils ne travaillaient; souvent mal nourris et peu soignés, ils ne faisaient pas l'objet d'un élevage, même s'ils avaient été produits quelque part, et leurs gènes se sont perdus sous l'effet des croisements et de l'introduction de races dûment identifiées. Ils participaient néanmoins à l'économie de l'élevage en fournissant un débouché, si maigre fût-il, aux autres étapes du commerce.

\section{Conclusion}

37 Chercher les réalités de l'élevage au XIX ${ }^{e}$ siècle, c'est donc se confronter à une abondance de sources écrites, pour certaines encore non identifiées, d'origines diverses, spécifiques au milieu du cheval ou non ; il s'agit de passer de descriptions d'ensemble à l'échelle d'un équidé précis, et de naviguer entre la compilation de séries considérables et l'analyse de données qualitatives détaillées, en s'appuyant sur des discours où le sens des mots dépend du positionnement ou de la personnalité de celui qui les emploie.

Alors que, du fait de l'omniprésence du cheval, son utilisation quotidienne ou l'action de faire saillir une jument semblaient à beaucoup de contemporains aller de soi, ces sources mettent en valeur les éleveurs qui s'affichaient comme tels à travers la participation à des concours ou l'entretien de reproducteurs, et qui ont fait les races, en cherchant la qualité. Le patrimoine écrit documente ainsi la constitution des patrimoines génétiques, l'émergence de groupes sociaux, la transmission de savoir-faire ou encore la construction d'imaginaires. Essentiel pour étudier l'articulation complexe entre élevage et travail, il contribue à souligner le rôle du cheval comme facteur de relations, tant moyen de transport qu'objet d'échanges commerciaux ou sujet de confrontation d'idées sur le beau et le bon, en théorie et dans la pratique.

\section{NOTES}

1. - La distinction entre pays d'élevage et de naissance a été magistralement définie par MUSSET, René. De l'élevage du cheval en France. Paris : Librairie agricole de la Maison rustique, 1917, p. 66.

2. - Voir FRÉMONT, Armand. L'élevage en Normandie, étude géographique. Caen : Faculté des lettres, 1967, vol. I, p. 12. DÉSERT, Gabriel. «L'élevage et les éleveurs de chevaux dans l'Orne sous le Second Empire ». Dans MANNEVILLE, Philippe, GENNES, Jean-Pierre de (dir.). Le cheval en Normandie. Actes du XXVIII ${ }^{e}$ congrès des sociétés historiques et archéologiques de Normandie, 
Mortagne-au-Perche, 21-24 octobre 1993. Caen: Musée de Normandie, coll. "Annales de Normandie, série des congrès des sociétés historiques et archéologiques de Normandie ", 1996, p. 37-50.

3. - Ce système perdura jusqu'à la fin du siècle, même si de moins en moins de demi-sang étaient utilisés aux travaux des champs: de nombreuses fermes les avaient remplacés par des chevaux de trait qui, moins fragiles, se dépréciaient moins en cas de blessure.

4. - Archives départementales de l'Orne, 1 ETP $428^{*}-435^{*}$ : Haras du Pin, registres de rapports sur la monte et la situation hippique en Basse-Normandie.

5. - Arch. dép. Orne, probablement 1 ETP 429*: Haras du Pin, rapport sur le service de la monte en 1854, 20 juillet 1854. Cet article se basant sur une consultation des archives du haras sur place, avant leur collecte par les archives départementales, les cotes sont estimées d'après l'inventaire. 6. - En particulier, des feuilles de monte remplies entre 1822 et 1827 ont été reliées; le palefrenier y inscrivait le signalement précis de chaque jument saillie, avec la date des revues, le nom et l'adresse du propriétaire.

7. - Il n'a pas été possible de déterminer quel auteur les a cités en premier ; l'affirmation a été reprise notamment par Charles Du Haÿs: DU HAŸS, Charles. Le cheval percheron: production, élevage, dégénérescence de la race, moyens de l'améliorer, foires importantes, principaux éleveurs, vitesse et tenue du percheron. Paris : Librairie agricole de la Maison rustique, 1866, p. 28. Voir le site : http://gallica.bnf.fr/ark:/12148/bpt6k165140p> [01/09/14].

8. - Arch. dép. Orne, probablement 1 ETP 387 et 388 : Haras du Pin, registres de matricules des étalons, 1809-1814 et 1814-1820. Les renseignements ont été trouvés dans un registre matricule concernant les étalons $\mathrm{n}^{\circ} 1$ à 187 ainsi que des juments, pouliches et poulains (1807-1834), et des registres de généalogie des étalons entrés entre 1805 et 1810 et entre 1810 et 1814 .

9. - En revanche, les habitudes des éleveurs ne permettent pas d'utiliser ces documents pour reconstituer des lignées avec certitude : il arrivait qu'une jument restée vide après une première saillie soit ensuite « revue " par un autre cheval de la même écurie, sans que cela soit inscrit. Le cas est rapporté par le directeur du haras du Pin pour l'étalonnier Desclos, de Saint-Ouen-deSècherouvre, dont l'étalon approuvé, vieux et fatigué, était remplacé pour les revues par l'un de ses trois autres chevaux, non approuvés. Arch. dép. Orne, probablement 1 ETP 431*: Haras du Pin, registre de rapports 1857-1861 : rapport sur la monte de 1859.

10. - Service historique de la Défense (SHD), $X^{S} 49$ à 52 et $X^{S} 171-172$; voir aussi fonds du général Préval, 1 M 1964-1967 ; Arch. dép. Orne, R 343.

11. - SHD, $X^{\mathrm{S}} 51$ : rapport spécial sur les opérations d'achat effectuées [...] pendant l'année 1863, dépôt de Caen. Voir aussi PERSON, Frédéric. Mémoires de la Société vétérinaire du Calvados et de la Manche : réponses aux questions du concours de 1849 (médaille d'or). Caen, 1849, p. 28-29 (publié aussi dans l'Argus des haras, 1850, tome 10, p. 400-481).

12. - Voir MARTIN, Jean-Claude. "Préfet et conseil général, deux acteurs du renouveau de l'élevage du cheval dans l'Orne sous le Premier Empire». Dans MANNEVILLE, Philippe, et GENNES, Jean-Pierre de, op. cit., p. 51-60.

13. - Voir MAYAUD, Jean-Luc. 150 ans d'excellence agricole en France. Histoire du Concours général agricole. Paris : Belfond, 1991.

14. - En pratique, il semble que des poulinières d'herbage ont pu y être admises, voire primées, à en croire des plaintes qui s'élevèrent notamment en 1881-1882, dont l'une fut publiée sous le titre "lettre d'un cultivateur» dans le Journal d'Alençon et du département de l'Orne, $\mathrm{n}^{\circ} 117$, samedi 7 octobre 1882. Voir aussi le compte rendu de la séance du conseil général du 23 août de la même année, où fut réaffirmé le fait que les juments « exclusivement consacrées à la reproduction et entretenues dans les herbages sans être jamais attelées " ne devaient pas aller au concours d'Écouché mais à celui du haras du Pin.

15. - Arch. dép. Orne, M 2975 et M 2076. 
16. - Les réponses aux enquêtes agricoles de 1852, 1862, 1882 et 1892 notamment nous apportent des renseignements très précis sur l'état de l'élevage et les structures agraires, même si leur interprétation demande de la prudence. Voir GARRIER, Gilbert. "Les enquêtes agricoles décennales au $\mathrm{XIX}^{\mathrm{e}}$ siècle: essai d'analyse critique». Dans Pour une histoire de la statistique (Journées d'études sur l'histoire de la statistique, Vaucresson, 23-25 juin 1976). Paris : INSEE/ Economica, 1977, tome 1: Contributions, p. 269-279.

17. - Arch. dép. Orne, 4 E 203/182 : minutes du notaire de Sainte-Scolasse-sur-Sarthe, inventaire après décès d'Alphonse Bignon, 15 avril 1880.

18. - Jument accompagnée de son poulain de l'année.

19. - Arch. dép. Orne, 4 E 203/183 : minutes du notaire de Sainte-Scolasse-sur-Sarthe, inventaire après décès de Rose Granger veuve Bignon, 7 et 14 décembre 1880 et 18 janvier 1881.

20. - Arch. dép. Orne, M 2022 : renseignements sur la production et le commerce des chevaux dans le département de l'Orne, donnés en 1860. Cette enquête fournit un indice a contrario en donnant comme explication à la vente de la quasi-totalité des poulains du canton de Vimoutiers le fait que cette proportion de un pour dix n'était pas même tolérée.

21. - « Rapport adressé au comice agricole de Putanges, le 5 mai 1853, par M. Hignard, vétérinaire à Écouché ». Dans Procès-verbal des séances du comice agricole de Putanges (Orne), fondé par M. le comte de Vigneral [...]. Argentan, 1853, p. 182-183.

22. - Extrait du « Bonhomme percheron » publié dans Journal d'Alençon, 6 décembre 1887, $\mathrm{n}^{\circ} 142$.

\section{RÉSUMÉS}

$\mathrm{Au} \mathrm{XIX}^{\mathrm{e}}$ siècle, l'élevage équin fut marqué par la mise en place de caractéristiques comme la définition de races spécialisées, l'émergence d'éleveurs reconnus et la forte présence de structures d'encadrement. À travers le cas d'un département emblématique, l'Orne, cet article s'attache à identifier les nombreuses sources, archivistiques et autres, produites autour des animaux de travail, et à étudier comment elles se complètent pour témoigner des réalités de l'élevage, de ses acteurs et de son environnement agricole ou commercial.

Workhorses in the nineteenth century, looking at horsebreeders in the Orne department. For horse breeding, the nineteenth century was a time of specialization, accompanied by the emergence of recognized breeders, and by supervision by various organizations. Through a case study of the department of Orne, reputed for its quality horse-breeding, the purpose of this article is to identify archive and other sources generated about work horses, and to examine how they complement each other and show the reality of breeding and its actors, within the farming and trading contexts.

INDEX

Mots-clés : élevage, XIXe siècle (1800-1899), archives, cheval, Basse-Normandie, haras, concours agricoles

Keywords : horse-breeding, nineteenth century, horse, stud farm, agricultural contest 


\section{AUTEUR}

\section{AGNÈS MANNEHEUT}

conservateur des bibliothèques, Université de Nantes agnes.manneheut@univ-nantes.fr 